\title{
Distal Aortic Remodeling after Type A Dissection Repair: An Ongoing Mirage
}

\author{
Kaushalendra Singh Rathore, F.R.A.C.S. \\ Department of Cardiothoracic Surgery, Sir Charles Gairdner Hospital, Nedlands, WA, Australia
}

\author{
ARTICLE INFO \\ Received April 12, 2021 \\ Revised May 29, 2021 \\ Accepted June 3, 2021 \\ Corresponding author \\ Kaushalendra Singh Rathore \\ Tel 61-8-6457-2383 \\ Fax 61-8-6457-2344 \\ E-mail kaushalendra.rathore@health. \\ wa.gov.au \\ ORCID \\ https://orcid.org/0000-0003-3183-1168
}

\begin{abstract}
Remodeling is a commonly encountered term in the field of cardiothoracic surgery that is often used to describe various pathophysiological changes in the dimension, structure, and function of various cardiac chambers, including the aorta. Stanford type A or DeBakey type 1 aortic dissection (TAAD) is a perplexing pathologic condition that can present surgical teams with the need to navigate a maze of complex decision-making. Ascending or hemi-arch replacement leaves behind a significant amount of distal diseased aortic tissue, which might have a persistent false lumen or primary or secondary intimal tears (or communications between lumina), which can lead to dilatation of the aortic arch. Unfavorable aortic remodeling is a major cause of distal aortic deterioration after the index surgery. Cardiac surgeons are aware of post-surgical cardiac chamber remodeling, but the concept of distal aortic remodeling is still idealized. The contemporary literature from established aortic centers supports aggressive management of the residual aortic pathology during the index surgery, and with continuing technical advancements, endovascular stenting options are readily available for patients with TAAD or for complicated type B aortic dissection cases. This review discusses the pathophysiology and treatment options for favorable distal aortic remodeling, as well as its impact on mid- to long-term outcomes following TAAD repair.
\end{abstract}

Keywords: Stents, Dissection, Intervention, Rupture

\section{Introduction}

Aortic dissection (AD) and aneurysm are end-stage eventualities of various aortic pathologies and are associated with significant morbidity and mortality if not treated promptly. AD has always captivated surgeons because of its fulminant course, and this constant engagement has brought improvement in contemporary surgical outcomes [1]. In $59 \%$ of patients with DeBakey type I or Stanford type A aortic dissection (TAAD), the entry tear is located in the proximal ascending aorta, while in other cases it might be found in the arch or distal aorta and can cause retrograde dissection along with malperfusion syndrome because of distal multiple re-entry tears (communications between lumina) $[2,3]$. Index proximal aorta surgery is reported to have good long-term results for TAAD repair, with a rate of freedom from proximal reoperation after aortic valve resuspension of $94.6 \%$ at 5 years and $76.8 \%$ at
10 years, and conservative hemiarch aortic replacement (HAR) is the most common surgical procedure performed [4]. However, it is often not sufficient to stabilize false lumen (FL) dimensions in the distal aorta, as it exposes the aorta for unfavorable remodeling, which eventually becomes a cause of distal secondary aortic intervention [3]. Ongoing unfavorable remodeling causes significant distal aortic dilatation ( $>55 \mathrm{~mm}$ or aortic growth of $10 \mathrm{~mm} / \mathrm{yr}$ ) and patients eventually undergo surgical or thoracic endovascular aortic repair (TEVAR) during follow-up [4]. These unwarranted morbidities during follow-up are the main reason for endorsing aggressive surgical resection during the index aortic surgery $[4,5]$.

\section{Defining remodeling}

Variability in reports is well recognized in the literature, and various groups have used different terminologies to 
describe similar phenomena. A European Cardiothoracic and Vascular Intervention Committee published a consensus document and suggested utilizing standard definitions for treatment and reporting purposes $[2,6]$. Remodeling is a process of stabilization of the overall aortic dimensions by slow obliteration of the FL and maintenance of true lumen (TL) patency. However, in the majority of the cases, this obliteration is incomplete and variable, which leads to aneurysmal dilatation of the aorta in $13.4 \%-62.5 \%$ of these patients after the index repair [7]. Aortic remodeling does not have an official definition, but it has been described by various surgical groups as favorable or unfavorable depending upon the occurrence of regression or progression with a $>10 \%$ volumetric difference in the aortic dimensions (TL, FL, or total aortic lumen) [8]. Other investigators have suggested using the aortic lumen diameter, as it correlates well with the cross-sectional area and could be used to assess aortic remodeling [9]. In most cases, favorable remodeling of the TL and FL is limited to the segment of the proximal thoracic aorta with the stent-graft in-situ, whereas volume changes of the TL and FL in the distal thoracic and abdominal aorta remain either stable or unpredictable $[7,8]$. Ulcer-like projections (ULPs) are examples of favorable remodeling seen in $62.3 \%$ of cases on computed tomography (CT) angiography scans [10]. Jang et al. [10] suggested that ULPs are healed intimal tears and thrombosed FLs or form at the detachment site of side branches and penetrating atherosclerotic ulcers. Intramural hematoma (IMH) is another sub-group of acute aortic syndrome that most often shows favorable remodeling and resolution in $50 \%-80 \%$ of cases, but in high-risk cases like elderly patients, those with an aortic diameter $>4.0 \mathrm{~cm}$, and those with an IMH thickness $>10 \mathrm{~mm}$ it might develop into dissection [11]. The current European Society of Cardiology guidelines define IMH as a circular or crescent-shaped thickening $>5 \mathrm{~mm}$ of the aortic wall with the absence of a dissecting membrane, intimal disruption, or FL flow [6]. Approximately $10 \%-30 \%$ of patients with acute aortic syndrome have associated IMH and most patients with IMH have Stanford type B dissection (50\%-85\%). Notably, IMH converts to acute aortic dissection in $88 \%$ of patients when the ascending aorta is involved, but in only $3 \%$ to $14 \%$ of cases when the descending aorta is involved. Patients with IMH have high rates of peri-aortic hematoma, pericardial effusion, and rupture in the mediastinum because it is located superficially on the aortic wall. Cardiac tamponade, which occurs in $19 \%$ of patients with acute type A dissection, is one of the most common causes of death in patients with IMH [12]. The echocardiographic criteria for diagnosing IMH include a wall thickness $>7 \mathrm{~mm}$ and an echolucent crescent-shaped zone in the aortic wall, which leads to compression of the aortic lumen [12]. It is often difficult to differentiate IMH from thrombosed FL and intimal calcification on various investigations such as echocardiography and cardiac-gated CT aortography. Magnetic resonance imaging can be used for the diagnosis and follow-up of patients with these findings.

Penetrating aortic ulcer (PAU) is an ulceration of an aortic atherosclerotic plaque penetrating through the internal elastic lamina into the media [6]. PAU is responsible for acute aortic syndromes in $2 \%-7 \%$ of cases and has a high rate of negative remodeling, which eventually leads to aortic rupture in $38 \%$ of cases. PAU is seen in the descending aorta in $94 \%$ of the patients while on the rest of the occasions seen in multiple locations in the aorta.

\section{Pathophysiology of patent false lumen or unfavorable remodeling}

The complete process of aortic dilatation or remodeling in $\mathrm{AD}$ is complex, multi-factorial, and incompletely understood. Interestingly, $>90 \%$ of patients presenting with acute TAAD would fail to meet the guidelines for elective ascending aortic replacement before dissection onset, and nearly two-thirds of non-marfanoid patients with spontaneous TAAD have a non-dilated ascending aorta before dissection onset [13]. Notably, however, during aortic dissection, the greatest increase in the diameter (30\%), cross-sectional area, tortuosity, ellipticity, and volume occurs in the ascending aorta [14].

Several reports have suggested that FL partial thrombosis, type A IMH, patency of the communication between lumina with a proximal location, greater FL thickness, initial aortic diameter, and the presence of ULPs are responsible for unfavorable remodeling $[11,13]$.

Among the various proposed theories, a widely accepted one is that high diastolic blood flow in the FL causes distension of the dissected chamber, especially in cases with a partially or completely thrombosed distal pouch. This leads to hemodynamic stress and structural weakening of the aortic wall, which might induce progressive enlargement of the affected aortic segment [15]. As explained earlier, obstruction of the FL drainage owing to blockage or the small size of the exit zones in the presence of continuous proximal filling is associated with pressurization of the $\mathrm{FL}$, resulting in the continuous expansion of the aorta. A crescent configuration of the TL in combination with a circular formation of the FL is suggestive of a large volume 
through the FL and had been held responsible for escalated aortic growth or unfavorable remodeling [16].

The most widely assessed criteria of remodeling are the TL, FL, and aortic lumen, which behave differently in different distal aortic segments following frozen elephant trunk (FET) or TEVAR. In the proximal thoracic aorta, both the FL and TL remodel positively, while in the abdominal aorta, the FL enlarges with time and the TL remains stable in size, while the aortic lumen increases because of the FL distension [17]. Lombardi et al. [17] divided the maximum trans-aortic diameter into 3 categoriesgrowth (increase of $>5 \mathrm{~mm}$ ), shrinkage (decrease of $>5$ $\mathrm{mm}$ ), or no change (increase or decrease within $5 \mathrm{~mm}$ ) and found that in the majority of the cases, the thoracic segment of the aorta was stable or shrunk $(80.3 \%$ at 1 year and $73.9 \%$ at 2 years).

\section{The false lumen, size of communications between lumina, and location}

Persistent proximal communications between lumina lead to elevated diastolic pressures in the FL (relative to those in the TL), resulting in delayed drainage with aortic diameter expansion and unfavorable remodeling in midterm follow-up [18]. Similarly, the proximity of these communications to the left subclavian artery (LSCA) and the number of tears are also important predictors of aneurysmal dilatation $[19,20]$. An FL and communications between lumina in the inner curvature of the aorta and tears at the concavity of the distal aortic arch are independent predictors of poor results [21].

Persistent filling of the FL is amongst the most common reasons for delayed or failed remodeling. A FL diameter of $22 \mathrm{~mm}$ or more in the proximal thoracic descending aorta was found to be an independent predictor for late aortic enlargement, and once the aortic diameter exceeds $60 \mathrm{~mm}$, the risk of FL rupture is around 30\% per year [22]. FL patency has been reported to be associated with a $20 \%$ reduction in patient survival after the index surgery and a $25 \%$ reduction in event-free survival [23]. An aortic cross-clamp may cause back-pressure in the dissected aortic wall, not only resulting in the complication of cerebral malperfusion, but also becoming a source of a persistent FL. A residual patent FL is the cause of unresected or secondary entry in the distal aorta. Although complete resection of all communication sites is required for thrombosis of the residual FL, the initial surgery for acute TAAD fails to achieve this objective, particularly in patients with primary or secondary entry located in the descending thoracic or abdominal aorta. Chronic FL patency is an independent factor to be associated with distal aortic enlargement; in order to decrease the incidence of residual patent FL, many authors have recommended systematic extended or total arch replacement during the index aortic surgery [3].

\section{Distal anastomotic leak}

A leak from a distal aortic-graft anastomosis is a common occurrence after hemiarch replacement, with a reported incidence between $39 \%$ and $70 \%$ [24]. Usually, anastomotic leak is diagnosed if an FL is visualized during the early phase of CT angiography, and another radiologic manifestation of pressurization of the FL is delayed emptying of the FL on late arterial-phase images. Almost all patients with accelerated distal aortic expansion $(>2 \mathrm{~mm} / \mathrm{yr})$ were found to have communications between lumina at the distal anastomosis, preventing complete thrombosis of the $\mathrm{FL}$ in all patients [25]. Another interesting observation is that incomplete thrombosis of the FL is more common when visceral branches arise from the FL, with the presence of the maximum diameter of the FL in the abdominal aorta and multiple communications between lumina [26]. Ueki et al. [26] demonstrated that FL thickness ( $>15 \mathrm{~mm}$ ), maximum descending aortic diameter, and the presence of a proximal communication between lumina were significant predictors of dissection-related events.

In approximately $70 \%$ of patients with $\mathrm{TAAD}$, the dissection flap extends beyond the ascending aorta and involves the aortic arch, and at least 1 entry tear was observed in $80 \%$ of patients on pre-discharge CT scans [27]. Rylski et al. [14] reported aortic growth following TAAD surgery where the distal dissected aorta was left behind and defined no aortic growth as a change of $<0.5 \mathrm{~mm} / \mathrm{yr}$, moderate aortic growth as $0.5-2.0 \mathrm{~mm} / \mathrm{yr}$, and accelerated aortic growth as $>2 \mathrm{~mm} / \mathrm{yr}$. Notably, they reported new entry tears during early follow-up in the proximal thoracic aorta (22\%), at the lesser curvature (11\%), and at the distal aorta-graft anastomosis (9\%). The largest increase in the total aortic lumen in their series was noticed $2 \mathrm{~cm}$ distal to the LSCA, reaching an average growth rate of $1.5 \mathrm{~mm} / \mathrm{yr}$, and it was influenced by the number of communications between lumina in the aortic arch, distal anastomosis leak, and persistent FL flow. A significant number of communications, especially in the aortic arch (36\%) and supra-aortic arteries (50\%), may compromise complete sealing of the entry tears during TEVAR and make it impossible to stop the blood flow through the FL.

The natural history of residual distal type B aortic dis- 
section (TBAD) after TAAD repair is different from that of primary TBAD, as the rate of interventions is significantly higher in primary TBAD [28]. In patients following TAAD repair, a patent primary entry tear is associated with a higher rate of interventions for the distal aorta, but this association is not found in patients with isolated TBAD. In chronic TBAD, the dissection flap and aortic wall become thick and less compliant, leading to incomplete remodeling even after TEVAR [29]. These cases require rigorous follow-up in early and mid-term follow-up. A variety of methods have been described to describe successful aortic remodeling after TEVAR such as evaluation of the FL/TL ratio, FL volumetry, and total aortic/aneurysm diameter changes over time on follow-up CT scans [30].

Saremi et al. [31] reported the following radiological predictors of unfavorable remodeling after TEVAR: a tortuous aorta with angulation, proximal endoleak, failure to cover proximal primary communications near the arch vessels, and thickened FL in chronic dissections. The etiology of persistent FL flow can either be from proximal aortic causes (distal suture line leak, unsealed landing zone of a stent, uncovered primary communications between intima, arch vessel leak, distal stent collapse, FL stenting, and defective stent) or from distal aortic causes (re-entry fenestration, intercostal arteries, fistula to pulmonary artery, an iatrogenic intimal tear, recurrent dissection, and an aberrant right subclavian artery).

Unfavorable remodeling in TAAD patients after supra-coronary ascending aorta replacement with aortic valve resuspension is well known, and De Paulis et al. [32] reported that at a 5 -year follow-up, $36 \%$ of patients presented with aortic root dilatation of $>10 \%$ and $56 \%$ of the patients progressed from mild aortic regurgitation (AR) to severe AR. These findings furnish evidence that negative aortic remodeling is a feature of diseased aortic tissue, and to increase event-free survival, extensive resection of the aortic tissue is mandatory-both proximally towards the root and distally towards the arch.

Another factor to mention is the detrimental effect of gelatin-resorcinol-formaldehyde (GFR) biologic glue (CryoLife Inc., Kennesaw, GA, USA), which has been reported by various groups to be a cause of unfavorable aortic remodeling that leads to pseudoaneurysm formation [31]. GFR bio-glue is known to cause macroscopic necrosis and microscopic acellular fibrosis with islands of hyaline material deposition $[32,33]$.

Stent-grafts and other endovascular interventions can be used to achieve favorable remodeling by aiding TF patency and by occluding FL backflow. There are various tech- niques to address these challenges from the TL side, such as TEVAR, PETTOCOAT (provisional extension to induce complete attachment), STABILISE (stent-assisted balloon-induced intimal disruption and relamination in aortic dissection repair), the Knickerbocker technique, branched stent-grafts, and the parallel stent-graft technique [6]. The benefits of trans-TL approaches are that they exclude entry tear in the proximal aorta and reduce flow through the FL, while simultaneously stabilizing the TL lumen.

However, there are few well-established techniques to achieve these goals through the FL, such as FL embolization and Candy Plug stent deployment [8]. Percutaneous balloon fenestration can be performed to create a tear in the intimal flap, helping in the free communication between the TL and FL [34]. Further, if the TL remains collapsed, TEVAR can be performed in the TL to increases TL perfusion pressure while simultaneously encouraging flow in the FL. The principal role of dissection flap fenestration is to help restore perfusion to branches compromised by dynamic obstruction. The advantages of trans-FL approaches are that they prevent excessive coverage of the distal aorta and reduce the chances of spinal cord ischemia.

\section{Impact of thoracic endovascular aortic repair on aortic remodeling}

Zhong et al. [35] reported that complete remodeling after retrograde TEVAR in the proximal descending aorta, distal thoracic aorta, and infra-renal abdominal aorta occurred in $89 \%, 78 \%$, and $50 \%$ of cases, respectively, during 31 months of follow-up. STABILISE is another technique to optimize the chances of abdominal remodeling by disrupting the FL and converting the aortic lumen into a single lumen. This technique increased the likelihood of complete remodeling in the infra-renal aorta compared to only TEVAR (82\% versus 12\%) [36]. However, in this technique, special attention is given to the aortic branches supplied by the $\mathrm{FL}$, and it is managed by visceral artery catheterization before inflation of the bare stent, and stenting of the side branches is required.

Heo et al. [37] described remodeling by dividing intimal tears into level 1 (between the distal anastomosis and the T7 vertebra), level 2 (T7 up to the coeliac trunk), and level 3 (below the coeliac trunk), and found a significant rate of aortic dilatation and interventions in patients with level 1 tears. The reason behind these changes might be high-pressure jet blood flow through the FL, leading to an increase in intraluminal tension and causing expansion of the FL 
chamber. Therefore, in these patients, aortic debranching and antegrade stenting should be performed. Although they reported faster favorable remodeling in the level 1 area following stenting, $21.4 \%$ of the patients still required re-interventions following TEVAR. All these reports underscore that remodeling rates are neither consistent nor favorably satisfactory in the distal aorta beyond the stentgraft-covered area $[38,39]$. The aortic lumen remains stable in acute and chronic dissections in the proximal aorta following TEVAR, irrespective of FL patency, but TL dimensions are strongly dependent on the FL patency. The external aortic wall (aortic lumen) has the ability to undergo remodeling even in the chronic phase, but TL remodeling is better only in acute dissections, which becomes a reason for re-intervention in the descending thoracic aorta in mid-term follow-up (69.5\%) [39].

The factors associated with secondary aortic interventions are device oversizing by $>10 \%$, bare-spring stents, early endoleaks with incomplete FL thrombosis, younger age, TAAD with a larger maximal aortic diameter at presentation, Marfan syndrome, and use of arch vessel adjunctive procedures with the index TEVAR. However, there was no survival difference at a 5-year follow-up in patients who received secondary aortic interventions. Giles et al. [39] demonstrated that secondary aortic interventions were a common feature following TEVAR for both acute and chronic TBAD patients. The major reasons described for TEVAR failure were persistent retrograde FL perfusion from distal re-entry tears, kinetic motion of the dissection flap and variable aortic-wall compliance, continued pressurization from the distal lumen despite thoracic FL aneurysm thrombosis, intimal-medial erosion from the stentgraft, endograft collapse due to a non-compliant dissection flap, and visceral vessel ischemia. Various forms of improvisation have been performed to correct these deficiencies of TEVAR in the distal thoraco-abdominal aorta. TEVAR covers proximal entry communications in the lumina, but leaves persistent perfusion in the distal FL, and therefore continues to have the potential for aneurysmal dilatation. Additional bare stent implantation in the thoraco-abdominal aorta has been proposed to stabilize the TL and intimal lamina (PETTICOAT technique). The PETTICOAT technique offers good short- and mid-term outcomes, but the FL is not completely sealed, and slow unfavorable remodeling continues. To avoid leakage between the stent and aortic walls, balloon-assisted full expansion of the covered and bare stent has been introduced (in the STABILISE technique), but this technique has the potential risk of aortic rupture during ballooning $[34,36]$.

\section{How to increase the chance of favorable remodeling following surgery?}

The review of the present literature strongly endorses aggressive resection of the distal diseased aortic segment and debranching of arch vessels with the deployment of FET to obliterate the distal FL (covering zones 0,1 , and 2 in the aorta) and keeping the TL open with stent support $[38,39]$. The conventional elephant trunk (ET) or FET and retrograde TEVAR are good options to improve unfavorable remodeling. Shrestha et al. [40] presented a 10-year follow-up and advocated FET rather than a conventional ET graft to prevent squashing of the distal TL diameter. They strongly suggested proper evaluation of intimal tears on preoperative scans to cover the proximal aorta completely and avoid endoleak.

The described average rate of early ( $<3$ months) FL thrombosis at the level of the stent-graft as assessed by imaging techniques is $85 \%$ (range, $69 \%-100 \%$ ). Moreover, it increases to $89 \%$ within 6-12 months after surgery, which confirms that aortic remodeling starts immediately after surgery and progresses over time [40]. FET helps TAAD patients in multiple ways. The first is immediate improvement by expanding and supporting the TL and excluding communications between lumina in the proximal aorta, thereby stopping malperfusion to the brain and visceral circulation. The second is by boosting favorable remodeling in mid- to long-term follow-up and reducing the requirement for further re-interventions. FET can also be used to remodel proximal aneurysmal dilatation following TAAD when there is no landing zone for retrograde TEVAR [41]. TEVAR in chronic TBAD is still known to cause retrograde TAAD by extending the FL proximally, and this iatrogenic negative remodeling might obscure the outcomes. In a recent cohort, most patients experiencing retrograde dissection after TEVAR had a primary communication at the concavity of the distal aortic arch [42]. The shape and diameter of the ascending aorta must be considered in the treatment of complicated type B dissections. The life-threatening situation of retrograde TAAD after TEVAR is caused by stent-related manipulation and the fragility of the entire aortic wall.

\section{Other adjunct modalities to help favorable remodeling are:}

(1) Many authors used angioscopy to better inspect the distal lumen to find intimal tears and for proper deploy- 
ment of the antegrade stenting, as a malpositioned stent might increase the chances of neurological events [43].

(2) The distal anastomosis can be performed using various buttress methods, such as applying a Teflon felt strip between the dissection layers (the sandwich technique) [37].

(3) Performing adventitial inversion into the TL can reduce the incidence of distal anastomotic leak and minimize surgical bleeding and distal malperfusion [44].

(4) "Neo-media" creation can be performed by inserting Teflon felt inside the dissected layers and fixation with bioglue [45].

\section{Predictors of distal aortic remodeling}

The contemporary literature indicates that an initial aortic diameter $>35 \mathrm{~mm}$, an FL diameter $22 \mathrm{~mm}$, and a large (>10 mm) proximal intimal tear are known risk factors for FL patency after conservative surgery (ascending aorta or hemiarch resection) for TAAD patients [46]. This accelerated aortic growth can be curtailed by complete resection of the diseased aortic segment, especially in young, stable patients with a large distal FL.

Exclusion of the proximal aortic tissue up to the orifice of the innominate artery (IA) and aggressive beveling of the lesser curvature of the arch extending distally to the level of the ligamentum arteriosum are the hallmarks of true hemiarch repair [47]. A group from Michigan Medicine reported that when dissected arch branch vessels without malperfusion syndrome were not repaired or replaced, there was no significant difference in postoperative stroke or perioperative mortality between hemiarch and arch replacement (zone 1/2/3) with the replacement of arch branch vessels [48]. They showed similar mid-term outcomes in both the hemiarch and debranching groups, but unsurprisingly, the re-intervention rate was higher in the conservative group (3.9\%/yr versus $2.2 \% / y r)$. These findings support an aggressive approach in stable and young patients with a dilated aortic arch, and most importantly, in the hands of an experienced surgeon.

Di Eusanio et al. [49] endorsed that younger patients with an intimal tear in the arch or distal aorta should be considered for total arch replacement instead of conservative HAR, as the freedom from re-operation rates at a 7 -year follow-up were $71 \%$ versus $85 \%$, respectively. The reasons for a persistent FL are incomplete resection of the primary intimal tear or the presence of unresected communications between lumina located in the arch or in the distal aorta. Di Eusanio et al. [49] also advocated using FET with debranching in younger patients with distal aor- tic malperfusion due to compression of the TL, complex primary re-entry tears involving the distal arch or proximal descending thoracic aorta, distal arch or descending thoracic aorta rupture, aneurysmal arch and proximal descending thoracic aorta, and a severely damaged aortic arch preventing a safe distal anastomosis.

A patent FL in descending aorta segments after the surgical treatment of TAAD is common (64\%-90\%). The principle of managing distal aortic malperfusion and improving the remodeling is obliterating the FL using graft extension (conventional frozen trunk or endovascular stenting using FET) and radial expansion of the TL with cardiac ejections. The most widely used hybrid endovascular prostheses are the Thora flex hybrid (Terumo Aortic, Sunrise, FL, USA) and the E-vita Open (JOTEC GmbH, Hechingen, Germany) [41]. Multiple reports have suggested that FET open stenting is far better than the conventional ET, and it is the preferred modality to manage complex DeBakey type I AD and aneurysms [3,5,35]. The conventional ET group had higher mortality, while the FET group had a higher frequency of complete remodeling (FL thrombosis rate, 64\% versus 29\%) and fewer distal aortic re-interventions [50].

The extension of the FL has been divided into 3 segments in relation to the stent-graft (the stented segment of the FET in the descending aorta is defined as segment A, the segment from the distal end of the stent-graft to the level of the coeliac trunk as segment B, and the segment from the coeliac trunk to the aortic bifurcation as segment C); this division is prognostic, as FL thrombosis is variable after FET deployment [6]. The incidence of persistent residual flow increases as the FL extends away from the stent-graft (90\% FL thrombosis at segment A and only 20\% at segment $\mathrm{C}$ or at the coeliac axis origin) [7]. Another reported advantage of the FET is that it reduces the rate of annual aortic growth in the covered part of the distal aorta, but does not stop dilatation in the distal aorta near the diaphragm and in the abdominal aorta, which eventually may lead to future aortic interventions [51]. Dohle et al. [8] reported their 10 years of experience with this technique and suggested that the FET technique enables 100\% avoidance of proximal endoleak and complications in the first half of the descending aorta using a circumferential suture line for FET fixation in the distal arch. Obliteration of the FL is also more frequent common in TAAD patients than in those with chronic dissections, especially in the distal thoracic aorta.

Partial thrombosis leads to a rise in the FL pressure, which increases wall tension and causes distal aortic aneu- 
rysmal changes with an eventuality of aortic rupture. Song et al. [52] reported that partial thrombosis of the FL is an independent risk factor for negative remodeling and is responsible for rapid distal aortic expansion, poor long-term survival, and aorta-related re-interventions after the index TAAD surgery.

Patients with connective tissue disorders (especially Marfan syndrome) are another important subgroup where distal aortic remodeling is poor, and these patients have a high aortic growth rate. Chen et al. [53] reported a series including 172 patients with Marfan syndrome and showed that the size of the distal aorta remained stable in $63.5 \%$ of patients at a follow-up of 6.2 years, and more importantly, disappearance of the FL with normal aortic segments was observed only in $28.8 \%$ of patients. They reported that the preoperative distal aortic maximum diameter $(>45 \mathrm{~mm})$ was an independent predictor of complications and reoperation during mid-term follow-up.

Evangelista et al. [54] reported the follow-up outcomes of 184 consecutive patients (108 surgically treated TAAD and 76 medically treated type B dissections) with patent FLs and reported that the presence of a large proximal entry tear was a predictor of mortality and required surgical/endovascular treatment in mid-term follow-up. The multivariate analysis in their series identified the baseline maximum descending aorta diameter, proximal location, and the size of the communication of lumina as predictors of dissection-related adverse events, whereas mortality was predicted by the baseline maximum descending aorta diameter, Marfan syndrome, and maximum descending aorta diameter. Another noticeable finding was a higher aortic growth rate in type $\mathrm{B}$ than in type A dissections (0.48 versus $0.21 \mathrm{~mm} / \mathrm{yr}$, respectively). A proximal descending aortic entry tear was seen in $44 \%$ of patients and was associated with poor outcomes, and most of these tears (68\%) were $10 \mathrm{~mm}$ or smaller.

Dohle et al. $[8,55]$ compared the effect of entries and exits on FL progression following TAAD repair using FET by dividing the entire descending aorta into 3 segments. Positive remodeling was found in segments A (94\%), B (63\%), and C (54\%), respectively, at a 1-year follow-up. The FL thrombosis quotients increased postoperatively across all 3 segments, and it was highest in segment A (98\%), followed by segments B and C (52\% and $28 \%$, respectively). They found negative remodeling in $>2$ segments in $43 \%$ of cases at the 1-year follow-up. Entries located in the proximal descending aorta and close to the distal anastomosis after isolated proximal repair had a poor impact on FL remodeling and mid-term outcomes.
However, these favorable findings should be taken with a grain of salt, as in $35 \%$ of the patients the FL remained complete or partially patent more distally, and endovascular stenting shifts FL patency-associated complications from the arch to the distal descending aorta [56].

\section{Frozen elephant trunk sizing to minimize unfavorable remodeling}

Antegrade stent sizing is paramount in minimizing endovascular leak, stabilizing the TL, and promoting aortic remodeling. Various groups have used different landmarks to size these grafts, but graft size is commonly determined at the level of the left atrium on preoperative cardiac-gated CT or echocardiography studies [57]. Intraoperatively, the point of reference for the positioning of the proximal end of the stent-graft is the cusp of the inner curve of the aortic arch. Hoffman et al. [57] supported using longer and bigger grafts that can cover the distal aorta up to the T10-T12 vertebrae, as it allows a more extensive repair of the dissected aorta, which limits the potential for residual type B dissection to a shorter distal section. Second, the total occlusion of the segmental arteries down to T10-T12 enhances the redistribution of spinal cord perfusion, which helps to develop collaterals and reduces the incidence of spinal cord ischemia during retrograde TEVAR.

Other groups have performed graft sizing based on the TL size and reported the incidence of FL patency to be between 5\% and 23\%. Uchida et al. [58] advocated intraoperative decision-making for FET sizing by inserting a ballshaped valve sizer into the TL of the descending aorta from a transverse incision in the arch under transesophageal ultrasound guidance. They strongly suggested correct sizing to avoid intimal tear in TAAD cases, especially in patients with Marfan syndrome.

Tsagakis and Jakob [59] suggested that the stent-graft size should be chosen according to the nature of the disease and the aortic diameter at the estimated distal landing zone. According to them, no oversizing in acute $\mathrm{AD}$, no oversizing or 1-size less in chronic $\mathrm{AD}$, and up to $10 \%$ over-sizing in aortic aneurysm should be done. In $\mathrm{AD}$, the maximal TL diameter is used for sizing. By placing the proximal FET anastomosis in arch position one 2 and using a stent-graft length of $130 \mathrm{~mm}$, the stented portion of the FET usually ends at descending aorta level T5. They also used intraoperative angioscopy to definitively determine the distal landing zone. In acute $\mathrm{AD}$, they achieved complete thrombosis of the FL in $90 \%$ of patients and $85 \%$ freedom from reintervention downstream at 8 years. Thus, 
FET induces positive aortic remodeling and eliminates residual dissection in the arch and descending aorta to a significant extent. Various groups have recommended moving the surgical field to zone 2 by performing debranching of the LSCA prior to the establishment of extra-corporeal circulation to minimize the deep circulatory hypothermic arrest period.

\section{Trade-offs against neurological complications}

Neurological complications are the most significant setback of these complex interventions and should be taken into consideration, as various surgical units have reported different stroke rates. A pooled analysis showed overall mortality of $8.8 \%$ in total arch replacement with FET deployment, where $53.2 \%$ of cases were done in emergency situations [60]. These single-digit mortality outcomes are because of data from high-volume specialized centers, which perform FET more often than conventional ET. Preventza et al. [60] presented a meta-analysis and reported an overall permanent or transient stroke rate of $7.6 \%$ in 32 studies, similar to what has been reported for conventional ET repair. However, this rate is higher than what was reported in previous FET meta-analyses, the reason for which could be the inclusion of only studies of total arch procedures, which are more complex and thus may incur a higher risk of stroke than proximal arch or hemiarch procedures. In their meta-analysis, they showed significantly higher rates of paralysis or paraparesis in patients with coverage at the T8 vertebra or beyond or a stent length of $15 \mathrm{~cm}$ or longer (spinal cord injury, 4.7\%; stroke, 7.6\%; operative mortality, 8.8\%). The spinal cord ischemia event rate was higher with a stent length of $15 \mathrm{~cm}$ or greater or coverage to $\mathrm{T} 8$ or beyond than with a stent length of $10 \mathrm{~cm}$ (11.6\% versus $2.5 \%$ ). Patients with TAAD and bovine arch-a common trunk of the IA and the left common carotid artery (LCCA) or an origin of the LCCA from the IA-were reported to have a $14 \%$ incidence of arch entry, which was significantly higher than that in patients without this pattern (59\% versus 13\%) and experienced a higher rate of postoperative neurological injury.

When a patent FL perfuses the thoraco-abdominal segment through multiple communications between the lumina, then it becomes a necessity for survival as it maintains visceral, renal, spinal, or peripheral perfusion. For this reason, it is a well-known aspect of the natural history of FL that distal runoff through these communications into the major vessels appears to be the mechanism of FL patency and the continued malperfusion of vital organs $[24,41]$.

\section{Conclusion}

These contemporary outcomes reemphasize the importance of complete resection or closure of any significant communications between the TL and FL in the distal ascending aorta. Favorable remodeling of the distal thoracic aorta following debranching with FET stent in complex TAAD or DeBakey type 1 dissections supports liberal but judicious usage of this technique in stable and young patients with minimum preoperative morbidity.

\section{Conflict of interest}

No potential conflict of interest relevant to this article was reported.

\section{ORCID}

Kaushalendra Singh Rathore:

https://orcid.org/0000-0003-3183-1168

\section{References}

1. Poon SS, Theologou T, Harrington D, Kuduvalli M, Oo A, Field M. Hemiarch versus total aortic arch replacement in acute type A dissection: a systematic review and meta-analysis. Ann Cardiothorac Surg 2016;5:156-73

2. Czerny M, Schmidli J, Adler S, et al. Current options and recommendations for the treatment of thoracic aortic pathologies involving the aortic arch: an expert consensus document of the European Association for Cardio-Thoracic surgery (EACTS) and the European Society for Vascular Surgery (ESVS). Eur J Cardiothorac Surg 2019;55:13362.

3. Sun L, Qi R, Chang Q, et al. Surgery for acute type A dissection with the tear in the descending aorta using a stented elephant trunk procedure. Ann Thorac Surg 2009;87:1177-80.

4. Geirsson A, Bavaria JE, Swarr D, et al. Fate of the residual distal and proximal aorta after acute type a dissection repair using a contemporary surgical reconstruction algorithm. Ann Thorac Surg 2007;84: 1955-64.

5. Fattori R, Cao P, De Rango P, et al. Interdisciplinary expert consensus document on management of type B aortic dissection. J Am Coll Cardiol 2013;61:1661-78

6. Rylski B, Pacini D, Beyersdorf F, et al. Standards of reporting in open and endovascular aortic surgery (STORAGE guidelines). Eur J Cardiothorac Surg 2019;56:10-20.

7. Zeng Z, Zhao Y, Wu M, et al. Endovascular strategies for post-dis- 
section aortic aneurysm (PDAA). J Cardiothorac Surg 2020;15:287.

8. Dohle DS, Tsagakis K, Janosi RA, et al. Aortic remodelling in aortic dissection after frozen elephant trunk. Eur J Cardiothorac Surg 2016;49:111-7.

9. Patterson BO, Vidal-Diez A, Karthikesalingam A, Holt PJ, Loftus IM, Thompson MM. Comparison of aortic diameter and area after endovascular treatment of aortic dissection. Ann Thorac Surg 2015;99:95-102.

10. Jang YM, Seo JB, Lee YK, et al. Newly developed ulcer-like projection (ULP) in aortic intramural haematoma on follow-up CT: is it different from the ULP seen on the initial CT? Clin Radiol 2008;63:201-6.

11. Alomari IB, Hamirani YS, Madera G, Tabe C, Akhtar N, Raizada V. Aortic intramural hematoma and its complications. Circulation 2014;129:711-6.

12. Pelzel JM, Braverman AC, Hirsch AT, Harris KM. International heterogeneity in diagnostic frequency and clinical outcomes of ascending aortic intramural hematoma. J Am Soc Echocardiogr 2007;20: 1260-8.

13. Rylski B, Branchetti E, Bavaria JE, et al. Modeling of predissection aortic size in acute type A dissection: more than $90 \%$ fail to meet the guidelines for elective ascending replacement. J Thorac Cardiovasc Surg 2014;148:944-8.

14. Rylski B, Blanke P, Beyersdorf F, et al. How does the ascending aorta geometry change when it dissects? J Am Coll Cardiol 2014;63: 1311-9.

15. Luebke T, Brunkwall J. Type B aortic dissection: a review of prognostic factors and meta-analysis of treatment options. Aorta (Stamford) 2014;2:265-78.

16. Tolenaar JL, van Keulen JW, Jonker FH, et al. Morphologic predictors of aortic dilatation in type B aortic dissection. J Vasc Surg 2013;58:1220-5

17. Lombardi JV, Cambria RP, Nienaber CA, et al. Aortic remodeling after endovascular treatment of complicated type $B$ aortic dissection with the use of a composite device design. J Vasc Surg 2014;59: 1544-54.

18. Tsai TT, Schlicht MS, Khanafer K, et al. Tear size and location impacts false lumen pressure in an ex vivo model of chronic type $B$ aortic dissection. J Vasc Surg 2008;47:844-51.

19. Tolenaar JL, van Keulen JW, Trimarchi S, et al. Number of entry tears is associated with aortic growth in type B dissections. Ann Thorac Surg 2013;96:39-42.

20. Sueyoshi E, Nagayama H, Hayashida T, Sakamoto I, Uetani M. Comparison of outcome in aortic dissection with single false lumen versus multiple false lumens: CT assessment. Radiology 2013;267: $368-75$.

21. Loewe C, Czerny M, Sodeck GH, et al. A new mechanism by which an acute type $B$ aortic dissection is primarily complicated, becomes complicated, or remains uncomplicated. Ann Thorac Surg 2012;93:
1215-22.

22. Song JM, Kim SD, Kim JH, et al. Long-term predictors of descending aorta aneurysmal change in patients with aortic dissection. J Am Coll Cardiol 2007;50:799-804.

23. Fattouch K, Sampognaro R, Navarra E, et al. Long-term results after repair of type $A$ acute aortic dissection according to false lumen patency. Ann Thorac Surg 2009;88:1244-50.

24. Rylski B, Hahn N, Beyersdorf F, et al. Fate of the dissected aortic arch after ascending replacement in type $A$ aortic dissection. Eur $\mathrm{J}$ Cardiothorac Surg 2017;51:1127-34.

25. Clough RE, Albayati MA, Donati T, Taylor PR. Uncomplicated type $B$ dissections: which patients should be treated?: lessons learned from the recent literature. J Cardiovasc Surg (Torino) 2014;55(2 Suppl 1):145-50

26. Ueki C, Sakaguchi G, Shimamoto T, Komiya T. Prognostic factors in patients with uncomplicated acute type B aortic dissection. Ann Thorac Surg 2014;97:767-73.

27. Conzelmann LO, Weigang E, Mehlhorn U, et al. Mortality in patients with acute aortic dissection type A: analysis of pre- and intraoperative risk factors from the German Registry for Acute Aortic Dissection Type A (GERAADA). Eur J Cardiothorac Surg 2016;49:e44-52.

28. Krahenbuhl E, Maksimovic S, Sodeck G, et al. What makes the difference between the natural course of a remaining type $B$ dissection after type $A$ repair and a primary type $B$ aortic dissection? Eur J Cardiothorac Surg 2012;41:e110-6.

29. VIRTUE Registry Investigators. Mid-term outcomes and aortic remodelling after thoracic endovascular repair for acute, subacute, and chronic aortic dissection: the VIRTUE Registry. Eur J Vasc Endovasc Surg 2014;48:363-71.

30. Stanley GA, Murphy EH, Knowles M, et al. Volumetric analysis of type $B$ aortic dissections treated with thoracic endovascular aortic repair. J Vasc Surg 2011;54:985-92.

31. Saremi F, Hassani C, Lin LM, et al. Image predictors of treatment outcome after thoracic aortic dissection repair. Radiographics 2018; 38:1949-72.

32. De Paulis R, Cetrano E, Moscarelli M, et al. Effects of ascending aorta replacement on aortic root dilatation. Eur J Cardiothorac Surg 2005;27:86-9.

33. Concistre G, Casali G, Santaniello E, et al. Reoperation after surgical correction of acute type A aortic dissection: risk factor analysis. Ann Thorac Surg 2012;93:450-5.

34. Khayat M, Cooper KJ, Khaja MS, Gandhi R, Bryce YC, Williams DM. Endovascular management of acute aortic dissection. Cardiovasc Diagn Ther 2018;8(Suppl 1):S97-107.

35. Zhong J, Osman A, Tingerides C, et al. Technique-based evaluation of clinical outcomes and aortic remodelling following TEVAR in acute and subacute type $B$ aortic dissection. Cardiovasc Intervent Radiol 2021;44:537-47.

36. Faure EM, El Batti S, Abou Rjeili M, Julia P, Alsac JM. Mid-term 
outcomes of stent assisted balloon induced intimal disruption and relamination in aortic dissection repair (STABILISE) in acute type $B$ aortic dissection. Eur J Vasc Endovasc Surg 2018;56:209-15.

37. Heo W, Song SW, Kim TH, et al. Differential impact of intimal tear location on aortic dilation and reintervention in acute type $I$ aortic dissection after total arch replacement. J Thorac Cardiovasc Surg 2019; 158:327-38.

38. Iafrancesco M, Goebel N, Mascaro J, et al. Aortic diameter remodelling after the frozen elephant trunk technique in aortic dissection: results from an international multicentre registry. Eur J Cardiothorac Surg 2017;52:310-8.

39. Giles KA, Beck AW, Lala S, et al. Implications of secondary aortic intervention after thoracic endovascular aortic repair for acute and chronic type B dissection. J Vasc Surg 2019;69:1367-78.

40. Shrestha M, Beckmann E, Krueger H, et al. The elephant trunk is freezing: the Hannover experience. J Thorac Cardiovasc Surg 2015; 149:1286-93.

41. Shrestha M, Bachet J, Bavaria J, et al. Current status and recommendations for use of the frozen elephant trunk technique: a position paper by the Vascular Domain of EACTS. Eur J Cardiothorac Surg 2015;47:759-69.

42. Gorlitzer M, Weiss G, Moidl R, et al. Repair of stent graft-induced retrograde type $A$ aortic dissection using the E-vita open prosthesis. Eur J Cardiothorac Surg 2012;42:566-70.

43. Dohle DS, Tsagakis K, Wendt D, et al. Angioscopy: a new intraoperative diagnostic and interventional tool for thoracic aortic treatment. Herz 2011;36:706-12.

44. Oda T, Minatoya K, Sasaki H, et al. Adventitial inversion technique for type $A$ aortic dissection distal anastomosis. J Thorac Cardiovasc Surg 2016;151:1340-5.

45. Leshnower BG, Chen EP. When and how to replace the aortic root in type A aortic dissection. Ann Cardiothorac Surg 2016;5:377-82.

46. Rylski B, Milewski RK, Bavaria JE, et al. Long-term results of aggressive hemiarch replacement in 534 patients with type $A$ aortic dissection. J Thorac Cardiovasc Surg 2014;148:2981-5.

47. Yang B, Norton EL, Shih T, et al. Late outcomes of strategic arch resection in acute type A aortic dissection. J Thorac Cardiovasc Surg 2019;157:1313-21.

48. Norton EL, Wu X, Farhat L, et al. Dissection of arch branches alone: an indication for aggressive arch management in type A dissection?
Ann Thorac Surg 2020;109:487-94.

49. Di Eusanio M, Berretta P, Cefarelli M, et al. Total arch replacement versus more conservative management in type $A$ acute aortic dissection. Ann Thorac Surg 2015;100:88-94.

50. Preventza O, Olive JK, Liao JL, et al. Acute type I aortic dissection with or without antegrade stent delivery: mid-term outcomes. J Thorac Cardiovasc Surg 2019;158:1273-81.

51. Sakaguchi G, Komiya T, Tamura N, et al. Patency of distal false lumen in acute dissection: extent of resection and prognosis. Interact Cardiovasc Thorac Surg 2007;6:204-7.

52. Song SW, Chang BC, Cho BK, et al. Effects of partial thrombosis on distal aorta after repair of acute DeBakey type I aortic dissection. J Thorac Cardiovasc Surg 2010;139:841-7.

53. Chen Y, Ma WG, Zhi AH, et al. Fate of distal aorta after frozen elephant trunk and total arch replacement for type $A$ aortic dissection in Marfan syndrome. J Thorac Cardiovasc Surg 2019;157:835-49.

54. Evangelista A, Salas A, Ribera A, et al. Long-term outcome of aortic dissection with patent false lumen: predictive role of entry tear size and location. Circulation 2012;125:3133-41.

55. Dohle DS, Jakob H, Schucht R, et al. The impact of entries and exits on false lumen thrombosis and aortic remodelling. Eur J Cardiothorac Surg 2017;52:508-15.

56. Tolenaar JL, Kern JA, Jonker FH, et al. Predictors of false lumen thrombosis in type $B$ aortic dissection treated with TEVAR. Ann Cardiothorac Surg 2014;3:255-63.

57. Hoffman A, Damberg AL, Schalte G, Mahnken AH, Raweh A, Autschbach R. Thoracic stent graft sizing for frozen elephant trunk repair in acute type A dissection. J Thorac Cardiovasc Surg 2013; 145:964-9.

58. Uchida N, Shibamura H, Katayama A, Shimada N, Sutoh M. Total arch replacement with an open stent graft for acute type $A$ aortic dissection: fate of the false lumen. Eur J Cardiothorac Surg 2009;35:838.

59. Tsagakis K, Jakob H. Which frozen elephant trunk offers the optimal solution?: reflections from Essen Group. Semin Thorac Cardiovasc Surg 2019;31:679-85.

60. Preventza O, Liao JL, Olive JK, et al. Neurologic complications after the frozen elephant trunk procedure: a meta-analysis of more than 3000 patients. J Thorac Cardiovasc Surg 2020;160:20-33. 